\title{
La formación universitaria como factor de riesgo para la ocurrencia de hostigamiento laboral en enfermería
}

\author{
Angelina M. Dois-Castellón
}

Introducción. El hostigamiento laboral en enfermería se ha posicionado como un problema de salud laboral relevante que afecta la dignidad de las personas y es fuente de desigualdad, estigmatización y conflicto. Su prevalencia varía entre el 17$87 \%$, pero estas cifras aumentan al considerar la victimización de la que son objeto los estudiantes, lo que podría contribuir a la incorporación de pautas violentas como parte de las relaciones habituales dentro de los equipos de enfermería.

Objetivo. Desvelar la relación entre el hostigamiento laboral y la formación universitaria desde la percepción de las enfermeras que han vivido experiencias de hostigamiento.

Sujetos y métodos. Diseño cualitativo, analítico-relacional. Los datos se recogieron a través de entrevistas semiestructuradas usando como criterio de finalización la saturación teórica y se analizaron según los procedimientos de la grounded theory.

Resultados. Los resultados muestran que la instrucción universitaria es un factor central en la forma en que las enfermeras establecen relaciones interpersonales y ejecutan su rol profesional, lo que serviría de base a la ocurrencia del hostigamiento laboral.

Conclusión. Debe asumirse un rol activo sobre aquellos aspectos relativos a la formación que reciben las estudiantes en las universidades y a las características de los servicios de enfermería donde desarrollan sus prácticas clínicas para erradicar las acciones hostigantes a las que se ven enfrentados los estudiantes, de forma que éstos puedan centrar su atención en el crecimiento profesional y no en la defensa de la propia integridad.

Palabras clave. Enfermería. Formación universitaria. Hostigamiento laboral.

\section{University education as a risk factor for the occurrence of workplace harassment in nursing}

Introduction. The nursing workplace harassment has been positioned as an important occupational health problem that affects the dignity of people and is a source of inequality, stigma and conflict. Its prevalence varies between $17 \%$ and $87 \%$, but these figures increase to consider the victimization of students who are subject, which could contribute to violent patterns incorporating as part of normal relationships within teams nursing.

Aim. To uncover the relationship between workplace harassment and university education from the perception of nurses who have experienced harassment.

Subjects and methods. Qualitative relational analytical design. Data were collected through semi-structured interviews using as completion criteria the theoretical saturation and were analyzed according to the procedures of the grounded theory.

Results. Results show that university education is a central factor in the way that nurses form relationships and perform their professional role which would serve as the basis for the occurrence of workplace harassment.

Conclusion. You should assume an active role on those aspects of training are available to students in universities and characteristics of nursing services where they develop their clinical practices to eliminate harassing actions to which students are confronted with so that they can focus on professional growth and not the defense of their own integrity.

Key words. Nursing. University education. Workplace harassment.

\section{Introducción}

El hostigamiento laboral en enfermería ha sido definido por el Consejo Internacional de Enfermeras y la Organización Mundial de la Salud, como incidentes donde el equipo es víctima de abuso, ame- nazas o agresión en circunstancias relacionadas con el trabajo y que involucra cambios implícitos o explícitos en la seguridad, bienestar o salud de los afectados $[1,2]$.

Estudios internacionales sugieren que el $25 \%$ de la violencia en el trabajo se dirige a trabajadores
Escuela de Enfermería. Facultad de Medicina. Pontificia Universidad Católica de Chile. Santiago, Chile.

Correspondencia:

Prof. Angelina María Dois Castellón. Escuela de Enfermería. Facultad de Medicina. Pontificia Universidad Católica de Chile. Avda. Vicuña Mackena, 4860. Macul, Santiago, Chile.

E-mail: adois@uc.cl

Conflicto de intereses: No declarado.

Competing interests: None declared.

(c) 2015 FEM 
de la salud y, de ellos, el personal de enfermería son las víctimas más frecuentes [3-5]. Esta situación ha sido verificada en distintos estudios dando cuenta de prevalencias que varían desde un $17 \%$ a un $87 \%$ [4,6-8].

De los factores relacionados con las causas del hostigamiento laboral en enfermería destacan los vinculados a la cultura organizacional de los equipos, que es adquirida, entre otras vías, por procesos de 'enculturación. Esto significa que el aprendizaje se logra a través de la observación de la forma de desarrollar el rol de otras enfermeras de mayor antigüedad y experiencia [5].

Los centros de práctica clínica se constituyen como lugares de integración de los contenidos teóricos recibidos en las universidades, pero también como los lugares donde los estudiantes comienzan a percibir las dinámicas de trabajo que ahí ocurren. Esta percepción influye directamente en su desarrollo profesional y en su capacidad de constituirse como profesionales autónomos, con capacidad resolutiva y con una actitud positiva hacia el cuidado $[9,10]$.

Sin embargo, en muchas ocasiones, estos lugares carecen del clima necesario para dicho fin e inciden de forma negativa en el aprendizaje de los estudiantes. Por ejemplo, en México, diversos estudios revelan que los estudiantes de enfermería opinan de forma desfavorable sobre el ambiente que encontraron en los servicios respecto a la percepción de respeto, aceptación, reconocimiento y confianza hacia ellos, con una alta frecuencia de conductas amenazantes repetidas durante su formación, entre las que se describen humillaciones frente a terceros, amenazas, gestos de intimidación e inculpación por faltas no cometidas, falta de reconocimiento y abuso dentro de las prácticas pedagógicas $[11,12]$. Situación similar fue la encontrada en Turquía, donde un estudio reveló que la totalidad del alumnado había sido víctima de violencia verbal perpetrada por compañeros, médicos, enfermeras y profesores, que se incrementaba a medida que avanzaban en su formación [13]. Por otro lado, resultados obtenidos en estudiantes de segundo y tercer año mostraron que más de la mitad había vivido o sido testigo de prácticas de hostigamiento hacia sus compañeros, que incluían humillaciones y abusos de poder por pares o personas en una posición jerárquica mayor [14].

En Chile no se cuenta con estudios en esta área. Por esta razón se busca desvelar la relación entre el hostigamiento laboral y factores relacionados con la formación universitaria desde la percepción de las enfermeras que han vivido experiencias de hostigamiento.

\section{Sujetos y métodos}

Estudio retrospectivo, analítico-relacional, cualitativo, basado en la grounded theory [15].

\section{Participantes}

La muestra inicial estuvo constituida por todas las enfermeras inscritas en las listas de correo electrónico del Colegio de Enfermeras de Chile. Para acceder a los sujetos de investigación se les envió una invitación abierta para participar en un estudio sobre dificultades interpersonales en el desempeño del trabajo. Las interesadas contestaron a una dirección electrónica, creada para fines de este estudio, y fueron contactadas telefónicamente para explicarles los objetivos de la investigación y el tipo de participación requerida. Para la selección de los participantes se intentó respetar al máximo los criterios de pertinencia, adecuación, conveniencia, oportunidad y disponibilidad para la obtención de los datos. De esta forma, los criterios de inclusión consideraron sólo la participación de enfermeras chilenas, formadas en universidades nacionales pertenecientes al Consejo de Rectores, con el fin de evitar que el fenómeno del hostigamiento se relacionara con discriminación por nacionalidad o pudiera atribuirse a alguna característica particular del contexto formativo si hubiera ocurrido en otro país. Además, debían identificarse como víctimas de hostigamiento laboral en el ejercicio de la profesión en instituciones de salud públicas o privadas, excluyendo situaciones de violencia física o acoso sexual y violencia de cualquier tipo ejercida por usuarios del sistema de salud. Debido a la dificultad para acceder a los sujetos de investigación, se utilizó muestreo por conveniencia y en cadena.

\section{Procedimiento}

Los datos se recogieron durante el período 2007 2008 usando entrevistas semiestructuradas mediante un guión de entrevista construido a partir de las preguntas directrices (Anexo). Este guión fue validado entrevistando a una enfermera que cumplía con los criterios de inclusión y modificado a la luz de esa entrevista piloto. Las entrevistas fueron grabadas, transcritas y analizadas en su totalidad utilizando como criterio de finalización la saturación teórica [15]. El análisis se realizó según los procedimientos de la grounded theory [15]. Para disminuir el sesgo del investigador, las categorías relevantes se determinaron según los resultados aportados por las entrevistas y los antecedentes bi- 
bliográficos. Además, se aseguró la validez y actualidad de los resultados triangulándolos con tres expertos en el tema (educación, currículo y disciplina) y dos de las enfermeras que en su momento participaron de las entrevistas.

La confidencialidad de la información, la autonomía de las participantes y el resguardo de su dignidad se aseguró a través del consentimiento informado aprobado para el proyecto de investigación n. 0702005010 (Purdue University Institucional Review Board).

\section{Resultados}

La muestra estuvo compuesta por 13 enfermeras formadas en universidades chilenas, que al momento de ser victimizadas tenían entre 23 y 56 años. La mayoría eran solteras, trabajaban en centros públicos y privados, de atención ambulatoria y hospitalaria y, en general, percibían sus salarios como relevantes para su manutención personal o de su familia. El promedio de desempeño en el cargo fue de 3,9 años, con un rango de 6 meses a 14 años, y la duración media del hostigamiento fue de 19 meses, con un máximo de 7 años. De los hostigadores, 11 eran mujeres y enfermeras y 9 ejercían cargos de jefatura.

El análisis descriptivo permitió identificar la construcción del rol profesional como categoría relacionada directamente con la visión de las enfermeras del fenómeno de hostigamiento laboral y que, a su vez, se vincularía estrechamente con la formación profesional recibida en las universidades a través de dos vías: la primera, relacionada con la formación en aspectos específicos de la profesión, y la segunda, con la formación en valores y actitudes de la enfermera.

De la formación específica en áreas de competencia profesional, las enfermeras describen una situación de indefensión técnica y profesional y falta de preparación, dada por una formación instrumentalizada, con bajo desarrollo del pensamiento crítico, déficit de habilidades para manejar situaciones de estrés y un quehacer profesional derivado del cumplimiento de indicaciones médicas: '... como que te crían con eso, que tú tienes que cumplir órdenes médicas, o sea que no, no puedes pasar más allá...' (E8, 137; las letras corresponden a las entrevistas y el número que acompaña a la letra corresponde al párrafo de la entrevista donde aparece la aseveración).

Por otro lado, la formación en actitudes y valores estaría marcada por una actitud de servicio, sumisión, tolerancia, estoicidad y autopostergación en función del cumplimiento del trabajo: '... creo que la victimización es una mala herencia de Florence Nightingale, no ves que Florence andaba con la lámpara, no tenía sueño, no se cansaba, no le pagaban, no le hacían... no, si esa es una idiotez de la enfermería...' (E2, 41).

Ambos aspectos, desde la percepción de las entrevistadas, influirían directamente en la claridad para la ejecución del rol profesional, lo que se evidenciaría en la inseguridad para desempeñar el trabajo, la poca visibilidad de éste y la falta de espíritu de grupo que se haría evidente a través de prácticas individualistas, rígidas, duras, fiscalizadoras y controladoras: '... creo que la enfermera no se ha preocupado de demostrar lo capaz que es, lo que es, lo que hace y cuál es su verdadero rol...' $(E 4,129)$.

A partir de los códigos obtenidos del análisis descriptivo se procedió con su análisis relacional, de lo cual se desprenden factores relacionados con la formación universitaria que contribuyen a la ocurrencia de fenómenos de hostigamiento laboral en enfermeras (Figura).

La formación profesional recibida en las universidades se vincula directamente, según las entrevistadas, con la construcción de aspectos específicos de la profesión y de los aspectos actitudinales asociados con ella, que a su vez se relacionan con la claridad con que la enfermera ejecuta su rol profesional y desarrolla su quehacer.

$\mathrm{Al}$ analizar las descripciones de las entrevistadas acerca de la formación recibida en las universidades, destaca que ésta se centraría en la tecnificación de la profesión y en la falta de desarrollo de un pensamiento crítico que les permitiera distinguir y relevar el foco de la disciplina. Más aún cuando, a juicio de ellas, ésta se encuentra altamente influida por una actitud de abnegación en el servicio a los demás, que permitiría responder a la imagen social colectiva con que se reconoce el rol de la enfermera, que deriva del cuidado 'religioso' y está supeditado a la acción del médico. En la misma línea, la formación que reciben las enfermeras se transformaría en un factor determinante para la formulación de su rol social, construyéndose así un círculo perpetuo y mutuamente reforzante.

Según las participantes, cada uno de los elementos descritos influiría en la forma en que la enfermera ejecuta su rol profesional, el cual podría seguir distintos patrones que dieran cuenta de la solidez de su construcción y de los paradigmas que ellas reconocen como propios y distintivos de la profesión. En esta ejecución, a juicio de las enfermeras, pueden distinguirse dos estilos conductuales: el primero, con una tendencia al ejercicio rígido del tra- 
Figura. Factores relacionados con la formación universitaria y la ocurrencia de hostigamiento laboral en enfermeras.

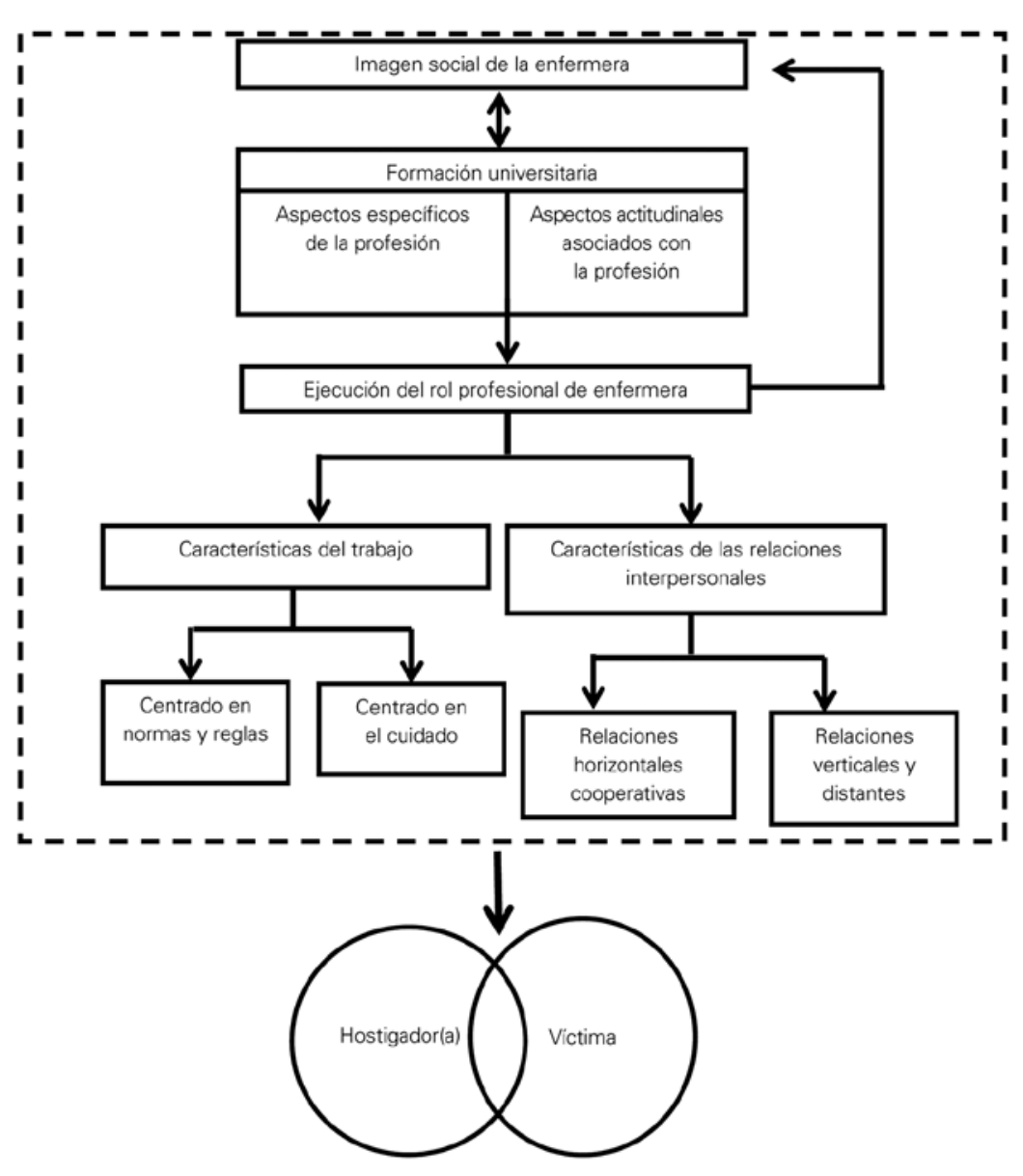

bajo, con limitaciones en la independencia y autonomía profesional que se reflejan en conductas apegadas a las normas y reglas vigentes y que dan cuenta de lo que ellas han definido como 'indefensión técnica y profesional'; el segundo se refiere a enfermeras que desarrollan su trabajo teniendo claro su rol y, por ello, son ejecutoras activas de un trabajo metódico en el que es posible distinguir el foco y esencia de la disciplina, como es el cuidado profesional.

En relación con estas dos tendencias observadas, las enfermeras distinguen algunas características personales que influirían en la forma de ejecutar el rol y en los estilos de relación interpersonal que establecen en el trabajo. Así, a su juicio, se pueden diferenciar dos patrones característicos en la forma de desarrollar el trabajo: uno, en el que se releva la sumisión, y otro que releva el autoritarismo y dos patrones de relación con los equipos de trabajo, el primero estableciendo relaciones horizontales de cooperación y cercanía, y el segundo en el que se distingue la verticalidad de las mismas, lo que las mantiene alejadas de sus pares. Todas estas características actuarían como facilitadores, mantenedores y perpetuadores del hostigamiento laboral.

\section{Discusión}

El hostigamiento laboral, por sus características y consecuencias, se ha considerado como una forma de estrés social grave que afecta tanto a la víctima como a la organización a la que pertenece $[16,17]$.

Se han descrito una serie de factores contextuales relacionados con su ocurrencia, entre ellos, su relación con la formación universitaria y su influencia sobre quienes están en proceso de formación, que luego se vincularía a la manera en que las enfermeras ejecutan el rol profesional durante la vida laboral. En relación a esto, las enfermeras describen que la formación profesional que reciben en los centros universitarios estaría fuertemente influida por la sobrevaloración de la técnica y la dificultad para desarrollar en los estudiantes actitudes que les permitieran posicionarse profesionalmente en el ejercicio del rol, entendiéndose éste como un rol ejecutado activa y autónomamente a partir de la reflexión crítica de las situaciones de enfermería, condición que desempeñaría un papel fundamental en la ocurrencia del hostigamiento laboral.

Si bien se han descrito algunas características en las enfermeras que hacen a unas más susceptibles de ser hostigadas y a otras más susceptibles de ejercer como hostigadoras, deben considerarse algunas condiciones que han sido estudiadas en los estudiantes de enfermería y que podrían ser la base para la construcción de un ser enfermera que preceda a la víctima y al hostigador potencial, aunque no puede aseverarse si responden a características constitutivas de quienes optan por estudiar la profesión o al moldeamiento de la persona durante la formación, considerando que los modelos profesionales que intervienen en su proceso educativo participan activamente en la construcción de su identidad como enfermeras y hacia la profesión [18], y que sea precisamente esta construcción la que facilite la ocurrencia y mantenimiento del hostigamiento laboral, ya sea desde la posición de víctima o de hostigador.

Las enfermeras participantes y la evidencia disponible describen que, durante el proceso de formación, muchas veces los alumnos se exponen a fenó- 
menos de hostigamiento por parte de los profesionales y docentes que participan en él, lo que se podría traducir en la incorporación de una pauta violenta de relación como modo habitual de desarrollar el trabajo, y que de alguna manera, invisibilizaría el fenómeno por considerarlo como constitutivo de la profesión. Ello se relaciona con los hallazgos de otros estudios respecto a cierto grado de aceptación de las situaciones violentas en los equipos de enfermería $[19,20]$. Este patrón relacional podría constituirse como parte de un currículo oculto (creencias, normas y valores sociales) que ejercería su influencia en la formación, aunque no aparezca dentro de los objetivos académicos, que se transmite eficazmente y se aprende a través de complejos mecanismos de imitación e interiorización, entre ellos, el proceso de modelaje de los estudiantes. Esto último puede relacionarse con el proceso de construcción de la identidad profesional y el mantenimiento de valores culturales asociados al género y al ejercicio del poder, incluyendo los asociados a las estructuras desiguales de poder y estatus [21-23].

Todas estas características podrían ejercer su influencia como elementos contextuales que estructurarían el marco sobre el cual se asentaría el fenómeno de hostigamiento laboral $[17,20,24]$.

En conclusión, el hostigamiento laboral en el personal de enfermería es un fenómeno de curso circular. Los elementos que intervienen en su desarrollo se relacionan recursivamente; no puede distinguirse una causalidad lineal, sino más bien un encadenamiento de factores que dan cuenta de la complejidad del fenómeno en sí.

Como docentes participantes en la formación de enfermeras no es posible abstenerse de esta realidad; más bien debe asumirse un rol activo para actuar sobre aquellos factores modificables, sobre todo en los aspectos relativos a la formación que reciben las estudiantes en las universidades y a las características de los servicios de enfermería donde se desarrolla su formación con el fin de erradicar las prácticas hostigantes en los equipos de enfermería docentes y asistenciales, lo que se traducirá en un mayor desarrollo de la profesión porque los estudiantes podrán centrar su atención en el crecimiento profesional y no en la defensa de la propia integridad.

Respecto a las limitaciones del estudio, decir que los resultados son el reflejo de la vivencia de hostigamiento laboral de un grupo particular de enfermeras y sus resultados, si bien comparten elementos con otros estudios en esta línea, no pueden ser generalizables. Además, la muestra estuvo formada sólo por mujeres, lo que no permite rescatar las experiencias de hostigamiento de hombres que se desempeñan como enfermeros. Por último, debido a las dificultades de acceso a los sujetos de investigación, el tipo de muestreo no cumplió con los criterios del muestreo teórico.

\section{Bibliografía}

1. Mayhew C, Chappell D. Workplace violence in the health sector: a case study in Australia. Journal of Occupational Health and Safety 2003; 19 (6) [internet]. URL: http://www. who.int/violence_injury_prevention/violence/en/wpv_ australia.pdf. [18.11.2011].

2. World Health Organization. Workplace violence. Geneva: WHO; 2005.

3. Amnistía Internacional. El cuidado de los derechos humanos: oportunidades y desafíos para el personal de enfermería y partería. Madrid: Amnistía Internacional; 2006.

4. Kwok R, Law Y, Li K, Ng Y, Cheung M, Fung V, et al. Prevalence of workplace violence against nurses in Hong Kong. Hong Kong Med J 2006; 12: 6-9.

5. Dois A. Hostigamiento laboral: una amenaza permanente para enfermería. Enfermería Global 2012; 28: 120-36 [internet]. URL: http://revistas.um.es/eglobal/article/view/eglobal. 11.4.143391. [16.01.2013].

6. Fornés J, Martínez-Abascal MA, García-Banda G. Variables socioprofesionales implicadas en el hostigamiento psicológico en enfermería. Enferm Clin 2008; 18: 127-33.

7. Uzun O. Perceptions and experiences of nurses in Turkey about verbal abuse in clinical settings. J Nurs Scholarsh 2003; 35: 81-5.

8. Wells J, Bowers L. How prevalent is violence towards nurses working in general hospitals in the UK? J Adv Nurs 2002; 39: 230-40.

9. Hong GY. Front-line care providers' professional worlds: the need for qualitative approaches to cultural interfaces. FQS 2001 [internet]. URL: http://www.qualitative-research. net/fqs-texte/3-01/3-01hong-e.htm. [16.01.2013].

10. Letelier, P, Valenzuela S. Violencia percibida por los alumnos de enfermería durante su formación clínica intrahospitalaria. Concepción: Universidad de Concepción; 2002.

11. Hernández G, Aguilar E. Condiciones del ambiente académicolaboral en la formación de estudiantes de enfermería. Rev Enferm IMSS 2003; 11: 125-32 [internet]. URL: http://www. medigraphic.com/pdfs/enfermeriaimss/eim-2003/eim033b. pdf. [10.02.2013].

12. Guillén $\mathrm{D}$. Violencia simbólica en la formación de estudiantes de enfermería. Desarrollo Cientif Enferm 2008; 16: 354-7 [internet]. URL: http://www.artemisaenlinea.org.mx/acervo/ pdf/desarrollo_cientifico_la_enfermeria/7ViolenciaSimbolica. pdf. [28.01.2013].

13. Celik S, Bayraktar N. A study of nursing student abuse in Turkey. J Nurs Educ 2004; 43: 330-6.

14. Curtis J, Bowen I, Reid A. You have no credibility: nursing students' experiences of horizontal violence. Nurse Educ Pract 2007; 7: 156-63.

15. Charmaz K. Grounded theory in the 21st century: applications for social justice studies. In Denzin N, Lincoln Y, eds. Handbook of qualitative research. 3 ed. Thousand Oaks, CA: Sage; 2005. p. 507-35.

16. Lewis M. Bullying in nursing. Nursing Standard 2001; 15: 39-42.

17. Quine L. Workplace bullying in NHS community trust: staff questionnaire survey. BMJ 1999; 318: 228-32.

18. Loo I. La identidad como proceso biológico-psicosocial y su construcción en enfermería. Revista Enfermería 2003; 11: 49-54.

19. Padilla T, Moreno E. El desarrollo profesional masculino: una perspectiva de género en la orientación profesional. Revista de Educación 2000; 2: 301-12.

20. Verde E, Gallardo G, Compeán S, Tamez S, Ortiz-Hernández L. 
Motivos de elección de carrera en mujeres estudiantes de profesiones de la salud. Educ Med 2007; 10: 44-51.

21. Hoel H, Giga S, Davidson M. Expectations and realities of student nurses' experiences of negative behaviour and bullying in clinical placement and the influences of socialization processes. Health Serv Manage Res 2007; 20: 270-8.

22. Royal College of Nursing. Working well: a call to employers [internet] 2002. URL: http://www.rcn.org.uk/members/ downloads/working_well_summary.pdf. [05.12.2011].
23. Mosteiro MJ. El género como factor condicionante de la elección de carrera: hacia una orientación para la igualdad de oportunidades entre los sexos. Revista Galego-Portuguesa de Psicoloxía e Educación 1997; 1: 305-15.

24. Hoel H, Cooper C. Destructive conflict and bullying at work. Manchester: Manchester School of Management, Institute of Science and Technology, University of Manchester; 2000.

\section{Anexo. Preguntas directrices y guión de entrevista}

\section{Preguntas directrices}

Considerando la evidencia disponible en relación a la ocurrencia de hostigamiento laboral en enfermería, se plantearon las siguientes preguntas directrices para intentar visualizar los factores involucrados en el hostigamiento laboral de las enfermeras y dar respuesta a los objetivos de la investigación:

1. ¿Qué elementos identifican las enfermeras como causales del desarrollo de las experiencias de hostigamiento laboral en enfermería?

2. ¿Qué características personales son descritas por las enfermeras como distintivas de las personas involucradas en relaciones de hostigamiento laboral en enfermería?

3. ¿Qué descripción hacen las enfermeras acerca de las características de las conductas de hostigamiento laboral en enfermería?

4. ¿Qué elementos identifican las enfermeras como intervinientes en las estrategias que utilizan para afrontar sus experiencias de hostigamiento laboral?

Guión de entrevista (las preguntas del guión se refieren a la situación de las entrevistadas al momento de vivir la experiencia de hostigamiento laboral)

1. Información demográfica
a. Pseudónimo
b. Edad
c. ¿Dónde trabaja? (hospital público/privado, clí- nica, consultorio, otro)
d. ¿Cuál es su cargo?
e. ¿Cuánto tiempo ha desempeñado ese cargo?

2. Datos referidos a situación de hostigamiento
a. ¿Que cargo o profesión tiene su jefe(a) direc- to(a)?

b. ¿Con quiénes debe trabajar? (cuántos, qué profesiones y cargos)

c. ¿Qué personas tiene a su cargo? (cuántas, qué profesiones y cargos)

d. ¿Cuál es su situación familiar? (con quién vive, número de hijos, estado civil)

e. ¿Cuán importantes son los ingresos que aporta a la economía familiar?

\section{Preguntas cualitativas}

a. Descripción de la situación

- ¿Podría explicar las dificultades interpersonales qué ha tenido en su trabajo?

- ¿Podría describir las experiencias que está viviendo? ¿Cuál es la más significativa? ¿Por qué?

- Comportamientos (suyos o de otros)

- Contextos

b. ¿Cómo ha ido evolucionando esta situación?

- ¿Cómo comenzó? ¿Recuerda algún momento particular?

- ¿Qué la ha hecho empeorar o, algunas veces, mejorar?

- ¿Qué está ocurriendo actualmente? Si fue en el pasado, ¿cómo se resolvió?

c. ¿Cómo siente que esta situación la ha afectado a usted?

- Físicamente

- Psicológicamente

- En relación con sus amigos y familiares

- Laboralmente

d. De todo lo que ha mencionado, ¿qué ha sido más difícil? ¿Qué otra cosa seguiría? ¿Hay algo más?

e. Las personas a veces usamos metáforas para describir nuestras experiencias. Por ejemplo, alguien puede describir sentimientos de felicidad diciendo que siente como si estuviera en las nubes. ¿Se le ocurre alguna metáfora 
para describir esta experiencia que está viviendo?

f. Saliéndonos de la metáfora, ¿cómo llamaría a la situación que está viviendo y observando? (por ejemplo, cuando alguien es ayudado por sus compañeros de trabajo, a eso se le puede llamar 'compañerismo')

- ¿Cómo cree usted que se le llama a estas experiencias?

- ¿Qué le parece este nombre?

- ¿Siente que esta palabra realmente representa lo que ha vivido (u observado)?

- Si pudiera cambiar el nombre o inventar uno nuevo para esta situación, ¿qué nombre usaría?

4. Dando cuenta de la experiencia

a. ¿Cómo se explica usted lo que le sucede?

b. ¿Por qué cree que le sucede esto a usted?

c. ¿Qué o quiénes cree que son culpables de que esto le ocurra?

d. ¿Ha visto que a otras personas en su trabajo les suceda lo mismo?

e. ¿Por qué cree que le pasó o les pasa?

f. ¿Qué o quiénes cree que son o fueron culpables de que esto ocurra?

g. ¿De qué manera estas experiencias difieren de la suya?

\section{Hostigamiento en enfermería}

a. En general, los datos muestran que las enfermeras son víctimas de hostigamiento laboral. ¿Por qué cree que se dan este tipo de situaciones (en su trabajo: consultorio, hospital, clínica, etc. y en el área de la salud)?

b. ¿Qué cree que facilita que se den este tipo de situaciones?

c. Si no se dan en su trabajo, ¿por qué cree que no se dan?

6. Manejando la experiencia

a. ¿Cómo ha manejado usted esta situación?

b. ¿Como describiría lo que ha hecho para manejar esta situación?

c. ¿Qué le gustaría hacer? ¿Por qué no lo ha hecho?

d. ¿Cree que hay algo que pudo haber hecho para evitar que esta situación ocurriera?

- Sí: ¿qué?

- No: ¿por qué? e. ¿Siente que está situación le ha sido más difícil de manejar que otras situaciones difíciles en su trabajo? ¿Por qué?

\section{Evaluando apoyo social y organizacional}

a. ¿Ha comentado esto con alguien? (familia, amigos, compañeros de trabajo, recursos humanos, sacerdote, médico)

b. ¿Con quién o quiénes?

c. ¿Cuáles han sido sus reacciones al escucharla?

d. ¿Qué le sugieren que haga?

e. ¿Cuánto le ha servido comentar esta experiencia con estas personas?

f. ¿Ha recibido algún apoyo concreto para afrontar esta experiencia? Por ejemplo, alguien le puede haber informado acerca de cómo funcionan los organismos de apoyo en el sistema de salud en que usted trabaja.

g. ¿De quién?

h. ¿En qué forma?

i. ¿Considera que el apoyo recibido representa una ayuda para superar esta situación? ¿Por qué?

j. En su trabajo, ¿cómo hubiera esperado usted que se manejara esta situación?

k. ¿En qué medida se han cumplido sus expectativas en este sentido?

1. ¿Qué le habría gustado que se hiciera de manera diferente?

m. Si existe hostigamiento en la actualidad, ¿cómo cree que podría resolverse esta situación para usted?

8. Comenzando a cerrar

a. Imagine que le dieran unos poderes mágicos para acabar con este tipo de situaciones en Chile, ¿qué es lo primero que haría?

b. ¿Qué cree que sería lo más eficiente para acabar con este tipo de situaciones?

c. ¿Hay algún aspecto de nuestra conversación que le gustaría ampliar? ¿Hay algo que le gustaría agregar o que yo no he cubierto y cree que sería importante para poder interpretar mejor sus respuestas?

d. A veces, al analizar entrevistas, uno se encuentra con aspectos que no comprende bien o que le gustaría explorar más. ¿Estaría dispuesta a que la contactáramos nuevamente para una segunda entrevista más corta?

9. Cierre. Expresión de gratitud 